\title{
Equilibrium Price Dispersion with Online Search
}

\author{
Lizhen $\mathrm{Xu}$ \\ McCombs School of Business \\ The University of Texas at Austin \\ lizhen.xu@phd.mccombs.utexas.edu
}

\author{
Jianqing Chen \\ Haskayne School of Business \\ The University of Calgary \\ jiachen@ucalgary.ca
}

\author{
Andrew Whinston \\ McCombs School of Business \\ The University of Texas at Austin \\ abw@uts.cc.utexas.edu
}

\begin{abstract}
This paper studies how consumers' online search behavior may trigger pervasive and persistent online price dispersion. We set up a game-theoretic model to examine the oligopolistic price competition, given the two unique features of online search, namely, the existence of a common search ordering and shoppers who have non-positive search cost. We show that in absence of further heterogeneity, the unique online search behaviors alone can drive significant level of price dispersion. Specifically, we derive twodimensional price dispersion, with both temporal fluctuation and spatial variation. We show that equilibrium price expectation monotonically decreases in line with consumers' search ordering. We also uncover a unique format of equilibrium pricing with stair-like sequence of price supports and localized price competition.
\end{abstract}

\section{Introduction}

Information technology has dramatically improved the efficiency of information sharing around the world. It greatly facilitates the search of useful information about products by significantly reducing the physical search cost. Meanwhile, thanks to the advance of ecommerce, almost all types of products can now be purchased online with several mouse clicks. Despite all these convenience brought by IT, an intriguing puzzle remains that the "Law of One Price" prediction has not realized with the development of the Internet and ecommerce. Price dispersion has been well observed as a pervasive and persistent phenomenon in the online environment. This work aims to add contributions to the theoretical explanations of online price dispersion.

Researchers have been devoting to exploring the driving forces of the prevalence and persistence of online price dispersion. Various theories emphasize the differentiation among products, from the actual differentiation in product quality or firms' competitive advantage to the virtual differentiation such as brand recognition. With the maturation of production technology and the standardization of operations process, firms converge quickly in terms of the quality level of products or services. Moreover, digitalization, one of the themes of the information era, not only greatly compacts possible space for product heterogeneity, but also diminishes potential competence difference among competing firms. Given the fact that the marginal production cost of digital product can be fairly low, if not zero, firms are facing head-to-head competition against numerous similar competitors (e.g., digital books from Ebook Library vs. NetLibrary, music from Napster vs. iTunes, and movies from Netflix vs. Hulu). In light of this, we take a different perspective and try to explain online price dispersion in absence of the heterogeneity among products and firms.

One classic view attributes price dispersion to the heterogeneity in consumers' search behavior. Compared to traditional off-line search, the Internet has revolutionized the way people search, largely owing to the powerful online search engines. In addition to providing relevant information to users' search queries, search engines are also able to gather numerous merchant sites competing in the same product market under the revolutionary advertising model---search advertising. In search advertising, by listing advertising links (or sponsored links) alongside search results for specific keywords according to advertisers' bids, search engines respond to potential consumers' queries by providing a list of merchant sites selling similar products which consumers are interested in. With its rapid growth rate, search advertising is predicted to become the leading advertising medium and the major commercial information provider (VSS, 2007), which attracts increasing research interest (e.g., Weber and Zheng, 2007; Xu et al., 2008).

Motivated by the unique features of consumers' online search behavior in association with search advertising, we study how these features affect the resulting price dispersion. Compared to traditional offline search, there are at least two distinctive features in consumers' online search behaviors: (i) There exists a commonly observed ordering; (ii) Consumers search 
costs are highly diversified, in particular, there exist "shoppers" who have non-positive search cost.

The first feature of online search behavior originates from the organization of advertisements in search engine result pages. The common format is that the sponsored links are listed on the right column alongside the organic search results, one after another from the top downward. Due to the reading habit and eye-movement pattern of most human beings, consumers usually process the information following the order of the list, from the top downward. Therefore, consumers generally first pay attention to the top advertising slot of the sponsored list, and then the next, and so on, while some of them stop searching in between. ${ }^{1}$ The arrangement of advertisements and the resulting ordering of the search create a huge prominence difference among advertising slots with different ranks. Empirical studies based on clickstream data have shown that the click-through rate attracted by the top link on a web page is generally the highest, and it decreases significantly from the top downward (e.g., Ghose and Yang, 2007).

The second feature owes to the advance of information technology, which greatly facilitates informational searches by significantly reducing the physical search cost. The physical cost to sample a product and quote the price from a store, which would otherwise be a non-negligible expense with necessary travel to the store, is now only several mouse clicks. In addition, some consumers do derive hedonic utility from shopping online (Childers et al., 2001): They enjoy the process of searching different places, comparing prices, and finding the best deal, evidenced by those who spend hours and hours surfing the web to shop. Altogether, with the flourishing of the Internet and online search engines, there arises a certain portion of consumers who have a non-positive (zero or even negative) net search cost. We call them shoppers. On the other hand, however, not everybody purchasing online has such luxury. The convenience of ecommerce brings many people with stringent time constraints, whose only goal is to find the product with a minimum of time spend. In addition, the information overload with the Internet and the extra skills needed to accomplish computer-based searches add to the cost for some online consumers. Therefore, there also exists a certain number of consumers who have a positive search cost, whom we may refer to as non-shoppers. ${ }^{2}$

\footnotetext{
${ }^{1}$ Hoque and Lohse (1999) use experimental data showing that compared with traditional paper media, consumers are more likely to pay more attention to the advertisements near the beginning of the heading in online directories.

${ }^{2}$ Early consumer research shows that a consumer's search effort is determined by various factors, such as time availability, purchase involvement, and attitudes toward shopping, and consumers do not
}

We set up a game-theoretic model, capturing the two features of consumers' online search behavior, to study how firms compete in price and the equilibrium price dispersion in search advertising environment. We consider oligopolistic competition in which multiple firms compete for consumers in a product market. Firms are differentiated in their ranks in consumers' search sequence. Consumers are assumed to follow a predetermined search ordering. They are differentiated in terms of search costs. In particular, there are a certain portion of consumers who have non-positive search cost and are willing to conduct thorough search. Other than the search ordering and search cost, we intentionally eliminate heterogeneity among firms and consumers in all other dimensions, so as to show that significant extent of price dispersion may arise even when identical firms sell identical products to identical consumers, and the driving force of such price dispersion is exactly consumers' unique online search behavior.

We derive a two-dimensional price dispersion pattern, reflecting the asymmetric mixed-strategy pricing equilibrium. In equilibrium, instead of statically charging one fixed price, firms randomize prices over particular ranges, which could result in variation of realized prices over time and accounts for the temporal price dispersion. Meanwhile, due to the asymmetric locational advantage, firms at different positions adopt different pricing strategies, which result in different price expectation across locations and accounts for the spatial price dispersion. We show that the temporal dispersion originates from the existence of shoppers, while the spatial dispersion is triggered by consumers' common search ordering. Furthermore, the equilibrium pricing derived from our model exhibits a unique and interesting format involving a stair-like sequence of price supports.

The major contribution of this work to the existing literature on search and pricing lies in that, to the best of our knowledge, we are the first to study the mixedstrategy equilibrium pricing in the setting of ordered search. Diamond (1971) raises the famous paradox that when consumers have positive search cost, an endogenous search model leads to a trivial equilibrium in which all firms charge monopoly price and consumers do not search. Varian (1980) suggests that when there exist consumers who are "informative" of all firms' prices, the equilibrium outcome may involve mixed-strategy pricing. Stahl (1989) studies how the existence of "shoppers" who have non-positive search cost and are aware of all firms' prices may affect the

always search thoroughly, even when purchasing expensive items (Beatty and Smith, 1987). Recent empirical investigation shows that online shoppers tend to search very few sites on average (Johnson et al., 2004). 
equilibrium pricing in a random search market. Since consumers are assumed to search randomly, there is no spatial price dispersion. In this paper, we consider shoppers in an ordered search market and derive richer price dispersion with the coexistence of both temporal and spatial pattern. Additionally, the unique format of equilibrium pricing derived from our model is absent in the previous literature.

Online price dispersion has been well documented in the previous literature, with evidences of both spatial and temporal price dispersion. For example, Clay et al (2001), Smith and Brynjolfsson (2001), and Chen and Hitt (2002) all show that significant levels of price dispersion exist online across different firms, even after control for various heterogeneities. Iyer and Pazgal (2003) and Baye et al (2004) find that the identities of the lowest-priced firms for various products online keep changing over time, which suggests a persistent level of temporal price dispersion.

The rest of the paper is organized as follows. In Section 2, we start with a model in which consumers search behavior is based on exogenous assumptions. Section 3 details the analysis and shows the main results. In Section 4, we extend the model to consider endogenous consumer sequential search decision. We show that similar results hold with endogenous search. Section 5 concludes the paper.

\section{Model}

There are $n \quad(\geq 2)$ firms selling homogeneous products and competing for consumers in a product market. These firms have a same marginal production cost, which is normalized to zero. There is a continuum of consumers with unit mass. Each consumer has a unit demand of the product and realizes a unit utility by consuming the product. Therefore, consumers will buy the product only if its price does not exceed 1 . Essentially, firms are identical except for their ranks in the search ordering, and consumers are identical except for their search behavior. By intentionally eliminating differentiation in all other dimensions, we are able to show that the unique features of consumers' online search behavior drive significant price dispersion even when identical firms sell identical products to identical consumers.

Consumers obtain product information through an online search engine, which lists hyperlinks directed to firms' websites where purchase can be conducted directly. Firms are placed at different positions in the list, which can be viewed as an outcome of pre-game competition such as bidding competition. ${ }^{3}$ Different positions have different prominence levels which can be strictly ordered. Without loss of generality, we call the most prominent position the first position, the second most prominent position the second one, and so on. For convenience, we call the firm at the $i$ th position firm $i(i=1, \ldots n)$. Consumers' search behavior is modeled in a way reflecting the two unique features of online search pattern: First, there exists a commonly observed search ordering so that all consumers start searching from the first position and may continue to the second, then the third, and so forth. Second, consumers' search costs are highly diversified so that they may stop the searching process at different stages. Especially, there exists a certain portion of shoppers, who have non-positive search cost, sampling all positions before making the purchase decision.

We start with the case in which consumers' sequential search decision is treated as exogenously given. Assume that after sampling the $i$ th position, a portion of $\alpha_{i}\left(0<\alpha_{i}<1\right)$ stops searching, while the other 1- $\alpha_{i}$ continue to sample the next position, such that the portion who visit the $i$ th $(i \geq 2)$ position is $\Pi_{j=1}^{i-1}\left(1-\alpha_{j}\right)$. To rule out violent fluctuation in the attention declining rates $\alpha_{i}^{\prime}$ s, we make the smoothness assumption that $\alpha_{i} \geq \alpha_{i+1}\left(1-\alpha_{i}\right) \quad(1 \leq i<n)$. This condition requires that the attention declining rates do not increase dramatically from one to the next, which can be easily satisfied (e.g., a same declining rate across positions $\left(\alpha_{i}=\alpha_{i+1}\right)$ satisfies this condition). In the extension, we endogenize consumers' search decision to show that similar results continue to hold.

The timing of the game is as follows. Firms first get placed at different positions in the search list. Based on their own positions, they price the product simultaneously. Consumers sample the position(s), learn the price(s), and make the purchase decision. For those who sample at least two positions, they purchase from the firm with the lowest price. When there is a tie in the lowest price, they randomly pick one firm with equal probability.

\section{Analysis and Results}

We first derive firms' equilibrium pricing strategies and then analyze the pattern of equilibrium price dispersion. In deriving the equilibrium pricing, first

\footnotetext{
${ }^{3}$ The bidding competition could also be included here. In fact, as they are identical before the pre-game competition, the firms adopt a same bidding strategies in equilibrium, and the equilibrium price dispersion remains the same.
} 
notice that due to the existence of shoppers, any static pricing is unstable.

Lemma 1: There is no pure-strategy equilibrium in the price competition.

Proof: Suppose there is a pure-strategy equilibrium in which firms price $\left\{p_{1}, p_{2}, \ldots, p_{n}\right\}$. If there is no tie in the lowest price, then it is profitable for the firm with the lowest price to deviate by increasing its price so that the price is closer to but still lower than the second lowest price. If there is a tie in the lowest price which is strictly positive, then any of the firms with the lowest price has profitable deviation by slightly cutting the price. If there is a tie in the lowest price which is zero, then among all these firms with zero price, the one at the best position can achieve positive profit by slightly increasing its price. Therefore, any pure strategy $\left\{p_{1}, p_{2}, \ldots, p_{n}\right\}$ cannot be an equilibrium.

Since there exists a certain portion $\left(\Pi_{j=1}^{n-1}\left(1-\alpha_{j}\right)\right)$ of consumers who sample all positions to look for the lowest price, a slight cut in price to become the lowest can lead to a significant increase in market share by capturing this portion of consumers. As a result, competing firms keep lowering their prices relative to the rivals. However, once the price is pushed to the lowest possible level (i.e., the competitive price), firms end in zero profitability. In this case, the firm at a better position in terms of the search ordering may deviate to achieve positive profit by charging a higher price and exploiting those consumers who stop searching right there. Therefore, any pricing strategy in which firms statically stay with one price cannot be stable. In other words, due to the presence of shoppers and the locational asymmetry created by the search ordering, it is not surprising that no pure-strategy equilibrium exists in the price competition.

Naturally, we next examine the mixed-strategy equilibrium pricing. We use $F_{i}(p), i=1, \ldots, n$, to describe firm $i$ 's mixed strategy of pricing. Like regular cumulative distribution functions, $F_{i}(p)$ measures the probability that firm $i$ charges a price less than or equal to $p$.

Proposition 1: The equilibrium mixed strategy of pricing from position $i$ is as follows. ${ }^{4}$

$$
\begin{aligned}
& F_{i}(p)=\left\{\begin{array}{ll}
1-\frac{\bar{p}_{i+1}}{p} & p \in\left[\bar{p}_{i+1}, \bar{p}_{i}\right) \\
1-\frac{\alpha_{i-1}\left(\bar{p}_{i-1}-p\right)}{\alpha_{i}\left(1-\alpha_{i-1}\right) p} & p \in\left[\bar{p}_{i}, \bar{p}_{i-1}\right]
\end{array} \quad(1 \leq i \leq n-1)\right. \\
& F_{n}(p)=1-\frac{\alpha_{n-1}\left(\bar{p}_{n-1}-p\right)}{\left(1-\alpha_{n-1}\right) p} \quad p \in\left[\bar{p}_{n}, \bar{p}_{n-1}\right]
\end{aligned}
$$

where $\bar{p}_{i}$ 's are recursively defined as

$$
\begin{aligned}
& \bar{p}_{0}=\bar{p}_{1}=1 \\
& \bar{p}_{i}=k_{i-1} \bar{p}_{i-1} \quad(i=2, . ., n)
\end{aligned}
$$

The coefficients $k_{i}$ 's are recursively defined as

$$
\begin{aligned}
& k_{n-1}=\alpha_{n-1} \\
& k_{i}=\frac{\alpha_{i}}{\alpha_{i}+\alpha_{i+1}\left(1-\alpha_{i}\right) k_{i+1}} \quad(i=n-2, \ldots, 1)
\end{aligned}
$$

Formal proof is detailed in the appendix. We use an example to illustrate the pattern of the equilibrium mixed-strategy pricing.

Example 1: Take a case of four positions with a same declining rate as an example. Specifically, let $n=4$ and $\alpha_{1}=\alpha_{2}=\alpha_{3}=1 / 2$. According to the above recursive definition in Eq.(3), $k_{3}=\alpha_{3}=1 / 2$, and it can then be derived $k_{2}=4 / 5$ and further $k_{1}=5 / 7$. Thus, according to Eq.(2), $\quad \bar{p}_{1}=1, \quad \bar{p}_{2}=k_{1} \bar{p}_{1}=5 / 7$, $\bar{p}_{3}=k_{2} \bar{p}_{2}=4 / 7$, and $\bar{p}_{4}=k_{3} \bar{p}_{3}=2 / 7$. Notice that by definition, the sequence of price support bounds $\left\{\bar{p}_{i}\right\}_{i=1}^{n}$ is monotonically decreasing, so that $\bar{p}_{1}>\bar{p}_{2}>\bar{p}_{3}>\bar{p}_{4}$. The pricing strategies of the four firms are as follows.

$$
\begin{aligned}
& F_{1}(p)= \begin{cases}1-\frac{5}{7 p} & p \in\left[\frac{5}{7}, 1\right) \\
1 & p=1\end{cases} \\
& F_{2}(p)= \begin{cases}1-\frac{4}{7 p} & p \in\left[\frac{4}{7}, \frac{5}{7}\right) \\
1-\frac{2(1-p)}{p} & p \in\left[\frac{5}{7}, 1\right]\end{cases} \\
& F_{3}(p)= \begin{cases}1-\frac{2}{7 p} & p \in\left[\frac{2}{7}, \frac{4}{7}\right) \\
1-\frac{2(5-7 p)}{7 p} & p \in\left[\frac{4}{7}, \frac{5}{7}\right]\end{cases} \\
& F_{4}(p)=1-\frac{4-7 p}{7 p} \quad p \in\left[\frac{2}{7}, \frac{4}{7}\right]
\end{aligned}
$$

Figure 1 illustrates the supports and distributions for the pricing strategies in these four positions.

In equilibrium, each firm achieves a constant expected payoff by charging any price within their price support. On the one hand, firm $i$ is willing to randomize price over its support $\left[\bar{p}_{i+1}, \bar{p}_{i-1}\right]$ because by pricing any price within the support, it achieves the same level of expected profit. On the other hand, indifferent between any prices within the support, firm $i$ mixes its prices in a way that gives its competitors a constant payoff within their price supports. Specifically, firms' expected profit functions can be written as

${ }^{4}$ To simplify the expression, define $\alpha_{0}=0$. 


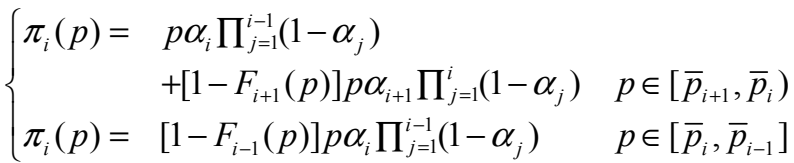

When firm $i(2 \leq i \leq n-2)$ charges a price within the lower half of its price support (i.e., $\left[\bar{p}_{i+1}, \bar{p}_{i}\right)$ ), among all consumers who sample its site (i.e., a portion of $\left.\Pi_{j=1}^{i-1}\left(1-\alpha_{j}\right)\right)$, it captures those who stop searching right there (i.e., a portion of $\alpha_{i} \Pi_{j=1}^{i-1}\left(1-\alpha_{j}\right)$ ), since its price is lower than firm $(i-1)$ 's price for sure (recall that

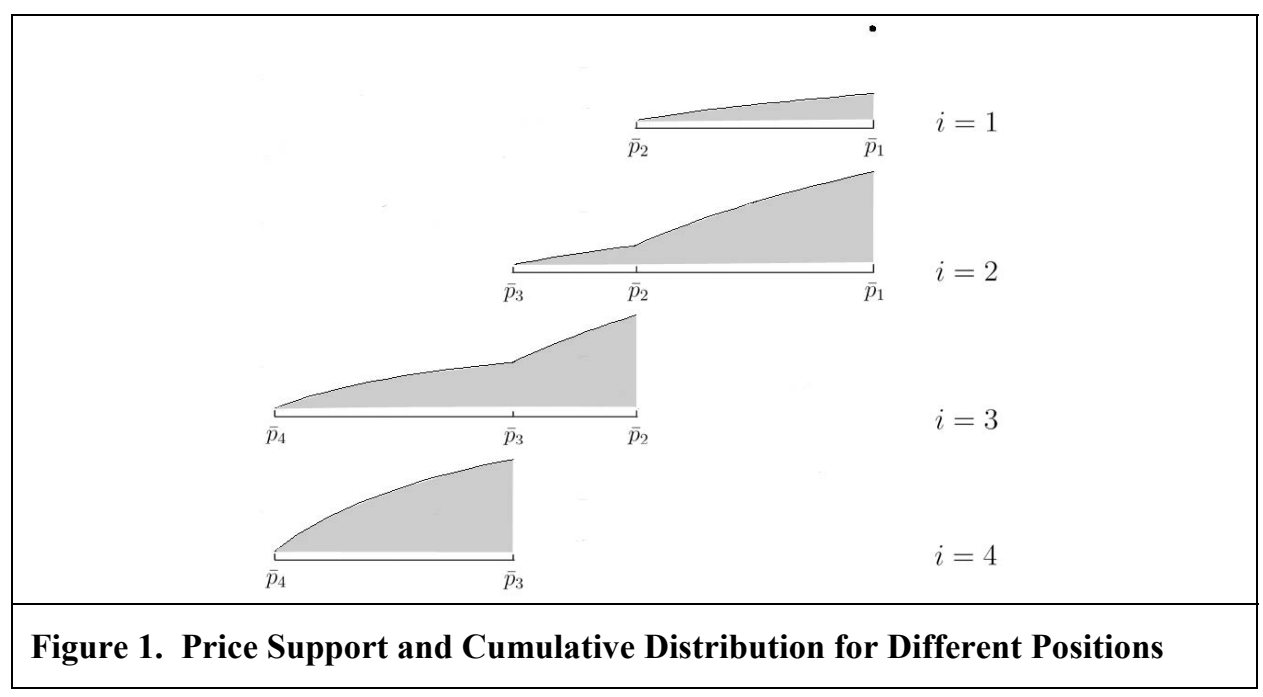

firm $i$-1's price support is $\left.\left[\bar{p}_{i}, \bar{p}_{i-2}\right]\right)$, which accounts for the first term of the first equation in Eq.(5). Firm $i$ can capture those who continue to sample the $(i+1)$ th position and stop there (i.e., a portion of $\left.\alpha_{i+1} \Pi_{j=1}^{i}\left(1-\alpha_{j}\right)\right)$ only when its price is lower than firm $(i+1)$ 's price, which accounts for the second term. Naturally, firm $i$ forgoes all those consumers who continue to sample the $(i+2)$ th position (i.e., a portion of $\left.\Pi_{j=1}^{i+1}\left(1-\alpha_{j}\right)\right)$, since firm $(i+2)$ 's price support is $\left[\bar{p}_{i+3}, \bar{p}_{i+1}\right]$ whose price is lower for sure. The second equation in Eq.(5), that is, the expected profit when firm $i$ prices within the upper half of its price support, can be interpreted in a similar way. Substituting in firms' equilibrium pricing strategies and by simple algebra, we can show that by charging any price within its strategy, firm $i$ achieves a constant expected profit $\alpha_{i} \prod_{j=1}^{i-1}\left(1-\alpha_{j}\right) \bar{p}_{i}$.

The next example illustrates the process how the equilibrium results can be derived, using the case of $n=2$.

Example 2: We consider the case of $n=2$ and denote $\alpha_{1}=\alpha$. First notice that any mixed-strategy equilibrium $\left\{F_{1}(\cdot), F_{2}(\cdot)\right\}$ should satisfy: (i) $F_{1}$ and $F_{2}$ have a common and continuous support $\left[\bar{p}_{2}, \bar{p}_{1}\right]$; (ii) neither $F_{1}$ nor $F_{2}$ has a mass point at the lower bound of the support, $\bar{p}_{2}$; (iii) $F_{1}$ and $F_{2}$ cannot both have a mass point at the upper bound $\bar{p}_{1}$. Next, we conjecture that $F_{1}$ has a mass point at the upper bound $\bar{p}_{1}$, which equals consumers' willingness-to-pay (i.e. 1). Thus, $\pi_{1}(p) \equiv \pi_{1}\left(\bar{p}_{1}\right)=\alpha \bar{p}_{1}$. To determine the lower bound $\bar{p}_{2}$, notice that $\pi_{1}\left(\bar{p}_{2}\right)=\bar{p}_{2}=\pi_{1}\left(\bar{p}_{1}\right)$, therefore, $\bar{p}_{2}=\alpha \bar{p}_{1} . \quad$ Thus,$\quad \pi_{2}(p) \equiv \pi_{2}\left(\bar{p}_{2}\right)=(1-\alpha) \alpha \bar{p}_{1} . \quad$ In equilibrium, both firms achieve constant payoff by pricing any price within the support. Therefore, by their payoff functions,

$$
\left\{\begin{array}{l}
\pi_{1}(p)=\alpha p+(1-\alpha) p\left[1-F_{2}(p)\right] \equiv \alpha \bar{p}_{1} \\
\pi_{2}(p)=(1-\alpha) p\left[1-F_{1}(p)\right] \equiv(1-\alpha) \alpha \bar{p}_{1}
\end{array},\right.
$$

we have the equilibrium pricing strategies

$$
\begin{aligned}
& F_{1}(p)= \begin{cases}1-\frac{\bar{p}_{2}}{p} & p \in\left[\bar{p}_{2}, \bar{p}_{1}\right) \\
1 & p=\bar{p}_{1}\end{cases} \\
& F_{2}(p)=1-\frac{\alpha\left(\bar{p}_{1}-p\right)}{(1-\alpha) p} \quad p \in\left[\bar{p}_{2}, \bar{p}_{1}\right]
\end{aligned}
$$

where $\bar{p}_{1}=1$ and $\bar{p}_{2}=\alpha \bar{p}_{1}$. We can verify that $F_{1}$ has a mass point at $\bar{p}_{1}$. We can also verify that it cannot be an equilibrium if $F_{2}$ has a mass point at $\bar{p}_{1}$.

Therefore, the equilibrium is unique by construction. 
An immediate corollary reveals the monotonic decrease of the expected profit of firms at different positions, which explains why the top position of a sponsored list in online search advertising is usually the most popular and engenders fierce bidding competition.

Corollary 1: Firms' equilibrium expected profits decrease monotonically from the first towards the last, i.e., $\pi_{i}>\pi_{i+1}, i=1, \ldots, n-1$.

Proof: Notice that $\pi_{i}=\alpha_{i} \prod_{j=1}^{i-1}\left(1-\alpha_{j}\right) \bar{p}_{i}$. Since $\bar{p}_{i}>\bar{p}_{i+1}$ and by the smoothness assumption $\alpha_{i} \geq \alpha_{i+1}\left(1-\alpha_{i}\right)$, therefore, $\pi_{i}>\pi_{i+1}$.

It is worth noting several features of the equilibrium pricing. First notice that except for the first firm, all firms' equilibrium pricing strategies are atomless within their entire support, including the upper and lower bound. The only mass point arises at the upper bound of the first firm's price support. This is because a mass point in one firm's price distribution would result in a downward jump of another firm's expected demand at that point and consequently lower profit levels in a contiguous region right to that point. For this reason, the only possible place where a mass point may occur is $\bar{p}_{1}$ : Although the mass point in $F_{1}(\cdot)$ causes a downward jump in firm 2 's expected profit at $p=\bar{p}_{1}$, since $F_{2}(\cdot)$ places non-positive probability measure on that particular point, firm 2's actual expected profit is not affected, which hence complies with the equilibrium requirement.

More interestingly, the sequence of the price supports here exhibits a stair-like shape. This is because firms have to take into account competition from both the firms ranked above and those ranked below. In fact, each firm only competes with its direct neighbors. They do not overcharge or undercut to compete with more distant opponents, because entering those territories only entangles itself into a more fierce competition which results in a less profit. This kind of stay-in-your-own-territory pattern keeps the price competition localized and sustainable, as can be observed in reality.

The mixed-strategy pricing means that, instead of statically charging one price, firms may charge any price within some possible price range according to certain probability distributions. If we imagine that consumers visit a particular position at different times, then the mixed pricing helps explain the existence of temporal price dispersion (i.e., the price varies over time, with occasional sales and frequent price fluctuations).
In addition to the temporal dispersion, noticing that firms at different positions adopt different pricing strategies, we are also interested to investigate the pattern of spatial price dispersion (i.e., (expected) prices vary across different locations). Intuitively, the firm at the first position will take advantage of the best location and charge the highest price. The next finding coincides with such expectation.

Proposition 2: The expected price decreases monotonically from the first position towards the last one, i.e., $E\left(p_{i}\right)>E\left(p_{i+1}\right), i=1, \ldots, n-1$.

Notice that firms next to each other adopt similar pricing strategies over the overlapped interval of their supports. In fact, according to Eq.(4), the conditional probability density functions of their pricing strategies over the overlapped interval are the same. In other words, $\quad E\left(p_{i} \mid p \in\left[\bar{p}_{i+1}, \bar{p}_{i}\right]\right)=E\left(p_{i+1} \mid p \in\left[\bar{p}_{i+1}, \bar{p}_{i}\right]\right)$. As firm $i$ is more likely to charge price within the upper half of its price support $\left(\left[\bar{p}_{i}, \bar{p}_{i-1}\right]\right)$, whereas firm $i+1$ also puts some probability mass in the lower half of the support $\left(\left[\bar{p}_{i+2}, \bar{p}_{i+1}\right]\right)$, it is not difficult to conclude that the unconditional expectation of firm $i$ 's price is strictly higher than that of firm $(i+1)$ 's.

Proposition 2 reveals an interesting pattern of spatial price dispersion, that is, the equilibrium price expectation decreases monotonically along the direction of consumers' search ordering. As a result, search is rewarding in the sense that those who keep searching are more likely to find a lower price. On the other hand, locational advantage is also rewarding in the sense that the firm at advantageous location may charge a price premium even with the same product.

Despite the fact that we have eliminated all potentially distractive differentiation among firms and consumers, we are able to derive two-dimensional price dispersion in equilibrium. It is thus clear that the unique features of consumers' online search behavior are among the fundamental driving forces of the pervasive and persistent price dispersion observed in the ecommerce environment. As we can see, due to the existence of shoppers with non-positive search cost, which is a direct result of the advance of information technology, firms price probabilistically rather than statically, which leads to the emergence of the temporal price dispersion. Meanwhile, due to the existence of a commonly observed search ordering, which is a prevalent phenomenon in online search advertising, firms with different ranks exploit their different levels of locational advantage and differ in the price expectation, which results in the appearance of spatial price dispersion. 


\section{Extension}

In this section, instead of treating consumers' sequential search decisions as exogenously given, we endogenize consumers' sequential search decisions. In other words, rather than assuming consumers stop searching at particular stages, we allow consumers to decide whether to conduct additional searches, given their heterogeneous search costs. As we will see, as long as there exists a commonly observed search ordering and a certain portion of shoppers with nonpositive search cost, a similar pattern of price dispersion continue to exist.

Following the model settings in the previous section, we now explicitly consider consumers' search costs. We focus on the case where consumers' search costs stay constant over the whole search process. Assume that consumer's search cost $s$ follows a certain distribution $G(s)$ over a support $[0, \bar{s}] . G(\cdot)$ has a mass point at the lower bound of the support, that is, there are certain portion of consumers with nonpositive search cost. We continue to assume that consumers search according to the given ordering, that is, they start from the first position, and continue sequentially towards the last one. Differently, consumers will conduct an additional search only if the expected gain from this additional search is not lower than the search cost.

We consider rational-expectations equilibrium. Consumers first form their search strategies; meanwhile, firms price their products; consumers then search according to their search strategies and make purchase decisions. For a profile of consumers' search strategies and firms' pricing strategy to be a rationalexpectations equilibrium, the following two conditions should be satisfied: First, given consumers' search strategies, firms have no profitable deviation in pricing; Second, given firms' pricing strategies, consumers' search strategies are rational.

For the simplicity of exposition, we consider $n=2$. Following similar reasoning as in the proof of Lemma 1 , it is not difficult to show that no pure-strategy equilibrium exists. We next characterize the mixedstrategy equilibrium.

For a consumer with search cost $s$, the complete search strategies include: the entry decision $\sigma(s)$, the reserve price from the first position $r(s)$ and the tiebreaking strategy $\lambda(s)$. The consumer enters the market and samples the first position with probability $\sigma(s)$; after the first sampling and learning the price $p$ from the first position, she stops searching if $p<r(s)$, continues to sample the second position if $p>r(s)$, and if $p=r(s)$, she stops searching with probability $\lambda(s)$. On the other hand, firms' pricing strategies can be characterized by the cumulative distribution functions, $F_{1}(\cdot)$ and $F_{2}(\cdot)$, over a common support $[\underline{p}, \bar{p}] .^{5}$

In any rational-expectations equilibrium, given firms' pricing strategies, consumers' search strategies should satisfy the following conditions. First, define the expected gain from sampling the first position, $E G_{1}$, as follows.

$$
E G_{1}=\int_{\underline{p}}^{\bar{p}}(1-x) d F_{1}(x)
$$

Thus, a consumer with search cost $s$ may conduct the first search only if the expected gain is greater than or equal to the search cost. In other words, $\sigma(s)=1$ if $s$ $<E G_{1} ; \sigma(s)=0$ if $s>E G_{1} ; \sigma(s) \in(0,1)$ only if $s=$ $E G_{1}$. Second, the reserve price $r(s)$ should satisfy

$$
s=\int_{\underline{p}}^{r}(r-x) d F_{2}(x)
$$

If we define the expected gain from the sampling the second position, after learning the price $p$ from the first position, as

$$
E G_{2}(p)=\int_{\underline{p}}^{p}(p-x) d F_{2}(x)
$$

then the condition specified by Eq.(7) is equivalent to requiring that a consumer should conduct an additional search only if $s \leq E G_{2}(p)$.

On the other hand, given consumers search strategies, firms at different positions simultaneously maximize their expected profits by setting the optimal pricing strategies. Denote $\hat{s} \in[0, \bar{s}]$ so that $\sigma(s)=1$ for $s \in[0, \hat{s})$ and $\sigma(s)=0$ for $s \in(\hat{s}, \bar{s}]$. Assume $F_{1}$ and $F_{2}$ are continuous within the support and $r(s)$ is continuous and monotonic so that its inverse function $s^{*}(p)$ exists. We can then formulate firms' profit functions. When $\bar{p}<r(\hat{s})$, for instance, their respective expected profits can be written as follows.

$$
\begin{aligned}
\pi_{1}(p)= & p \delta(\hat{s}) \sigma(\hat{s})+p\left[G(\hat{s})-\delta(\hat{s})-G\left(s^{*}(p)\right)\right] \\
& +p \delta\left(s^{*}(p)\right) \lambda\left(s^{*}(p)\right) \\
& +p\left[1-F_{2}(p)\right]\left[G\left(s^{*}(p)\right)-\delta\left(s^{*}(p)\right) \lambda\left(s^{*}(p)\right)\right]
\end{aligned}
$$

$$
\pi_{2}(p)=p \int_{p}^{\bar{p}}\left[G\left(s^{*}(x)\right)-\delta\left(s^{*}(x)\right) \lambda\left(s^{*}(x)\right)\right] d F_{1}(x)
$$

where $\delta(\cdot)$ measures the possible point mass in the search cost distribution $G$. The first three terms in Eq.(9) represent firm 1's profit from those captured consumers who stop sampling the second position. The

\footnotetext{
${ }^{5}$ As we can show, firms' equilibrium pricing strategies should have a common support.
} 
last term in Eq.(9) represents the expected profit from those sample both positions. Notice that the partition of consumers' search behavior is now endogenously determined by firm 1's price, which directly affects the possible demand of the second firm, as by Eq.(10). Firms' profit functions under other price range can be formulated in a similar fashion. In equilibrium, by substituting in $F_{2}(\bar{p})=1$ and $F_{2}(p)=0, \bar{p}$ solves the optimization problem $\max _{\bar{p}} \pi_{1}(\bar{p})$, and $\underline{p}$ can be defined by $\pi_{1}(\underline{p})=\pi_{1}(\bar{p})$. In consequence, the equilibrium pricing strategies can be obtained by solving $\pi_{i}(p)=\pi_{i}(p)$.

With certain specific forms of search cost distribution $G$, we are able to derive closed-form equilibrium pricing and further investigate the pattern of equilibrium price dispersion. Next, we consider a simple form of such distribution, $\tilde{G}$, which is defined as

$$
\tilde{G}(s)=\left\{\begin{array}{c}
1-\alpha s \in[0, k) \\
1 \quad s=k
\end{array}\right.
$$

In other words, among all consumers, 1- $\alpha$ of them are shoppers with non-positive search cost, while the other $\alpha$ have positive search cost $k(>0)$ and may be referred as non-shoppers. By explicitly solving the pricing equilibrium under this distribution, we examine the resulting price dispersion pattern with endogenous sequential search decision. As we can see, similar pattern of two-dimensional price dispersion can be observed.

Proposition 3: When $\alpha(1-\ln \alpha) r \leq 1-k$, where $r=\min \left\{\frac{1-\alpha}{1-\alpha+\alpha \ln \alpha} k, 1\right\},\left\{\sigma(s), r(s), \lambda(s) ; F_{1}(p), F_{2}(p)\right\}$ is a rational-expectations equilibrium, in which $\sigma(0)$ $=\sigma(k)=1, r(0)=0, r(k)=r, \lambda(0)=0, \lambda(k)=1$, and $F_{1}$ and $F_{2}$ are defined as follows.

$$
\begin{aligned}
& F_{1}(p)= \begin{cases}1-\frac{\alpha r}{p} & p \in[\alpha r, r) \\
1 & p=r\end{cases} \\
& F_{2}(p)=1-\frac{\alpha(r-p)}{(1-\alpha) p}
\end{aligned}
$$

In the equilibrium specified by Proposition 3 , the firm at the first position charges reasonable prices so that the expected gain from the first sampling (by Eq.(6)) exceeds non-shoppers' search cost $k$. As a result, all consumers enter the market (i.e., $\sigma(0)=\sigma(k)$ $=1)$. Not surprisingly, since shoppers have nonpositive search cost, it is optimal for them to keep searching. In that sense, they set their reserve price at the lowest possible price (i.e., $r(0)=0$ ) and continue to conduct an additional even when they are indifferent (i.e., $\lambda(0)=0$ ). In contrast, non-shoppers have a much higher reserve price due to their higher search cost (i.e., $r(k)=r$ ). They stop searching if the expected gain from an additional search (by Eq.(8)) does not exceed their search cost (i.e., $\lambda(k)=1$ ). Anticipating consumers' search behaviors, the first firm sets the upper bound of its price support equal to non-shoppers' reserve price $r$, in order to retain these portion of consumers from further searching. Meanwhile, given consumers' search strategies and firm 1's pricing strategy, the firm at the second position can achieve profit only by competing for shoppers with relatively lower price. Similar as before, $F_{1}$ and $F_{2}$ are so set that both firms attain constant payoff by charging any price within the entire price support. Also notice that $F_{1}$ has a mass point at the upper bound, while except for that point, both firms' price distributions are atomless.

Notice that by endogenizing consumers' sequential search decision, we observe similar pattern of temporal price dispersion. In fact, the equilibrium price distributions in Eq.(13) share the same form as those in Eq.(1). Further investigation shows that a similar pattern of spatial price dispersion can be observed.

Proposition 4: The equilibrium price expectation decreases with the search ordering, i.e., $E\left(p_{1}\right)>E\left(p_{2}\right)$.

It is also worth discussing the case when the parametric condition in Proposition 3 (i.e., $\alpha(1-\ln \alpha) r \leq 1-k)$ does not hold. It occurs when $\alpha$ is relatively large, that is, most consumers have reasonably high search cost. In that case, if all of these non-shoppers enter the market with $\sigma(k)=1$, the firm at the first position cannot resist charging them high prices to fully exploit these large portion of demand. As a result, the expected gain from the first sampling $E G_{1}$ can be even lower than non-shoppers' search cost $k$, which contradicts their rational decisions. In equilibrium, instead of entering the market deterministically, non-shoppers adopt probabilistic entry decision so that $\sigma(k) \in(0,1)$ and $E G_{1}=k$. The following numerical example illustrates the equilibrium pricing in this type of scenario.

Example 3: Let $\alpha=4 / 5$ and $k=1 / 5,\{\sigma(s), r(s), \lambda(s)$; $\left.F_{1}(p), F_{2}(p)\right\}$ is a rational-expectations equilibrium such that $\sigma(0)=1, \sigma(k)=\beta, r(0)=0, r(k)=r, \lambda(0)=$ $0, \lambda(k)=1$, and $F_{1}$ and $F_{2}$ are defined as

$$
\begin{aligned}
& F_{1}(p)= \begin{cases}1-\frac{\alpha \beta r}{(1-\alpha+\alpha \beta) p} & p \in\left[\frac{\alpha \beta}{1-\alpha+\alpha \beta} r, r\right) \\
1 & p=r\end{cases} \\
& F_{2}(p)=1-\frac{\alpha \beta(r-p)}{(1-\alpha) p} \quad p \in\left[\frac{\alpha \beta}{1-\alpha+\alpha \beta} r, r\right]
\end{aligned}
$$

where $\{r, \beta\}$ solves the system of equations 


$$
\left\{\begin{array}{r}
r-\frac{\alpha \beta}{1-\alpha} \ln \frac{1-\alpha+\alpha \beta}{\alpha \beta} r=k \\
1-\frac{\alpha \beta}{1-\alpha+\alpha \beta}\left(1+\ln \frac{1-\alpha+\alpha \beta}{\alpha \beta}\right) r=k
\end{array}\right.
$$

Numerically, $r=0.88$ and $\beta=0.39$. We can further compute the expected prices from both positions. $E\left(p_{1}\right)$ $=0.80$ and $E\left(p_{2}\right)=0.68$. Similar as before, $E\left(p_{1}\right)>$ $E\left(p_{2}\right)$.

When considering consumers' endogenous sequential search decision, firms have to take into account consumers' possible reaction to the pricing strategies they may adopt. In light of this, instead of taking guaranteed demand for grant and fully exploiting them, firms have to adjust their prices to cater to consumers' search strategies so that they are able to retain certain portion of consumers from further searching. Nevertheless, since the relative locational advantage among firms, which is originated from the search ordering, remains the same, as firms adjust their pricing strategies simultaneously, the relative spatial pattern of prices across different positions remains unchanged. Moreover, with shoppers searching around, firms have to randomize their prices to maintain sustainable profit, which engenders temporal price fluctuation. As a result, even when endogenizing consumer search decision, as long as a search ordering and shoppers still present, a similar pattern of twodimensional price dispersion continues to hold.

\section{Conclusion}

This paper studies how consumers' online search behavior may trigger pervasive and persistent online price dispersion. We set up a game-theoretic model to examine the oligopolistic price competition, given the two unique features of online search, namely, the existence of a common search ordering and shoppers who have non-positive search cost. Firms are differentiated only in their rank in consumers' search sequence, whereas consumers are differentiated only in their search costs. We show that in absence of further heterogeneity, the unique online search behaviors alone can drive significant level of price dispersion. Specifically, we derive two-dimensional price dispersion, with both temporal fluctuation and spatial variation. We show that equilibrium price expectation monotonically decreases in line with consumers' search ordering, which implies that searching is rewarding and a prominent location could be profitable in general. We also uncover a unique format of equilibrium pricing with stair-like sequence of price supports and localized price competition. By considering shoppers in an ordered search market, we contribute to the literature by studying asymmetric mixed-strategy pricing in oligopolistic competition.

\section{Appendix}

\section{Proof of Proposition 1}

Denote $F_{i}(p)$ as $F_{i}^{-}(p)$ for $p \in\left[\bar{p}_{i+1}, \bar{p}_{i}\right)$ and $F_{i}^{+}(p)$ for $p \in\left[\bar{p}_{i}, \bar{p}_{i-1}\right]$.

(i) We can show that $F_{i}(\cdot), i=1, \ldots, n$, is well defined cumulative distribution function. First notice that all supports are well defined since $0<k_{i}<1$, $i=1, \ldots, n-1$, and thus $\left\{\bar{p}_{i}\right\}_{i=1}^{n}$ is positive and monotonically decreasing. For each $F_{i}^{-}(\cdot)$ or $F_{i}^{+}(\cdot)$, it is strictly increasing within its support; $F_{i}^{-}\left(\bar{p}_{i}\right)=F_{i}^{+}\left(\bar{p}_{i}\right) \quad$ because $\quad 1-F_{i}^{-}\left(\bar{p}_{i}\right)=\frac{\bar{p}_{i+1}}{\bar{p}_{i}}=k_{i}$ $=\frac{\alpha_{i-1}}{\alpha_{i}\left(1-\alpha_{i-1}\right)}\left(\frac{1}{k_{i-1}}-1\right)=\frac{\alpha_{i-1}}{\alpha_{i}\left(1-\alpha_{i-1}\right)} \frac{\left(\bar{p}_{i-1}-\bar{p}_{i}\right)}{\bar{p}_{i}}=1-F_{i}^{+}\left(\bar{p}_{i}\right)$.

Therefore, each $F_{i}(\cdot)$ is strictly increasing in its entire support. Since $F_{i}\left(\bar{p}_{i+1}\right)=0$ and $F_{i}\left(\bar{p}_{i-1}\right)=1, F_{i}(\cdot)$ is well defined.

(ii) Next, we show that each position $i$ yields a constant expected profit $\pi_{i}$ within the entire support $\left[\bar{p}_{i+1}, \bar{p}_{i-1}\right]$. If the firm in position $i(i=2, \ldots, n-1)$ prices at $p \in\left[\bar{p}_{i}, \bar{p}_{i-1}\right]$, it only competes with the firm in position $i-1$ for the demand $\alpha_{i} \prod_{j=1}^{i-1}\left(1-\alpha_{j}\right)$. Thus, the expected profit in the upper interval will be

$$
\pi_{i}^{+}(p)=\left[1-F_{i-1}^{-}(p)\right] \cdot p \cdot \alpha_{i} \prod_{j=1}^{i-1}\left(1-\alpha_{j}\right) \equiv \alpha_{i} \prod_{j=1}^{i-1}\left(1-\alpha_{j}\right) \bar{p}_{i} \text {. }
$$

If it prices in the lower interval $\left[\bar{p}_{i+1}, \bar{p}_{i}\right]$, it will win over the firm above and capture the demand $\alpha_{i} \prod_{j=1}^{i-1}\left(1-\alpha_{j}\right)$, and meanwhile compete with the firm below for the demand $\alpha_{i+1} \prod_{j=1}^{i}\left(1-\alpha_{j}\right)$, which leads to the expected profit

$$
\begin{aligned}
\pi_{i}^{-}(p) & =p \alpha_{i} \prod_{j=1}^{i-1}\left(1-\alpha_{j}\right)+\left[1-F_{i+1}^{+}(p)\right] p \alpha_{i+1} \prod_{j=1}^{i}\left(1-\alpha_{j}\right) \\
& \equiv \alpha_{i} \prod_{j=1}^{i-1}\left(1-\alpha_{j}\right) \bar{p}_{i} .
\end{aligned}
$$

Therefore, $\pi_{i}^{+}(p)=\pi_{i}^{-}(p)$, i.e., the expected profit remains constant over the entire support.

For the firm at position $n$, it competes with the firm above it only. A calculation similar to that for the above $\pi_{i}^{+}(p)$ shows a constant expected profit $\prod_{j=1}^{n-1}\left(1-\alpha_{j}\right) \bar{p}_{n}$. Similarly, we can verify the constant expected profit for the firm at position $1 .^{6}$

${ }^{6}$ The only exception is that there will be a downward jump in 
(iii) Now we verify that no unilateral profitable deviation exists by pricing outside the given support. Suppose the firm in position $i(\geq 3)$ prices at $p>\bar{p}_{i-1}$. If $p<\bar{p}_{i-2}$, it faces a competition with the two firms above and the expected profit is

$$
\begin{aligned}
& \pi_{i}(p)=\left[1-F_{i-1}^{+}(p)\right]\left[1-F_{i-2}^{-}(p)\right] \cdot p \cdot \alpha_{i} \prod_{j=1}^{i-1}\left(1-\alpha_{j}\right) \\
& =\alpha_{i} \prod_{j=1}^{i-1}\left(1-\alpha_{j}\right) \frac{\alpha_{i-2}\left(\bar{p}_{i-2}-p\right)}{\alpha_{i-1}\left(1-\alpha_{i-2}\right)} \frac{\bar{p}_{i-1}}{p}
\end{aligned}
$$

which is strictly decreasing in $p$, i.e., $\pi_{i}(p)<\pi_{i}\left(\bar{p}_{i-1}\right), \forall p \in\left(\bar{p}_{i-1}, \bar{p}_{i-2}\right)$. If $p \geq \bar{p}_{i-2}$, then the firm would have zero demand (and thus zero profit) since the firm right above charge a lower price for sure. For the firms at position 1 and 2, they will clearly not charge a price higher than $\bar{p}_{1}$, since $\bar{p}_{1}=1$, consumers' willingness-to-pay. Thus, there is no unilateral profitable deviation by pricing higher beyond the given support.

Suppose the firm in position $i(\leq n-2)$ prices at $p<\bar{p}_{i+1}$. If $p \in\left[\bar{p}_{i^{\prime}+1}, \bar{p}_{i^{\prime}}\right]\left(i^{\prime} \geq i+1\right)$, it could capture all consumers who stop sampling at any position from $i$ to $i^{\prime}-1$. We denote the amount of those consumers as $D_{i i^{\prime}}$. In addition, it competes with the firm on position $i^{\prime}$ and $i^{\prime}+1$ for consumers who stop sampling at position $i^{\prime}$ and $i^{\prime}+1$. Thus, the expected profit can be written as

$$
\begin{aligned}
\pi_{i}(p) & =D_{i i^{\prime}} p+\left[1-F_{i^{\prime}}^{-}(p)\right] p \alpha_{i^{\prime}} \prod_{j=1}^{i^{\prime}-1}\left(1-\alpha_{j}\right) \\
& +\left[1-F_{i^{\prime}}^{-}(p)\right]\left[1-F_{i^{\prime}+1}^{+}(p)\right] p \alpha_{i^{\prime}+1} \prod_{j=1}^{i^{\prime}}\left(1-\alpha_{j}\right) \\
& =D_{i i^{\prime}} p+\left[1-F_{i^{\prime}}^{-}(p)\right] \cdot \alpha_{i^{\prime}} \prod_{j=1}^{i^{\prime}-1}\left(1-\alpha_{j}\right) \bar{p}_{i^{\prime}} \\
& =D_{i i^{\prime}} p+\alpha_{i^{\prime}} \prod_{j=1}^{i^{\prime}-1}\left(1-\alpha_{j}\right) \bar{p}_{i^{\prime}} \frac{\bar{p}_{i^{\prime}+1}}{p}
\end{aligned}
$$

where the second and third steps are by substituting $F_{i^{\prime}+1}^{+}(p)$ and $F_{i^{\prime}}^{-}(p)$ in, respectively. Note that $D_{i i^{\prime}} \geq \alpha_{i^{\prime}-1} \prod_{j=1}^{i^{\prime}-2}\left(1-\alpha_{j}\right) \geq \alpha_{i^{\prime}} \prod_{j=1}^{i^{\prime}-1}\left(1-\alpha_{j}\right)$, where the last inequality is due to the smoothness assumption. Therefore, $\pi_{i}\left(\bar{p}_{i^{\prime}}\right)-\pi_{i}\left(\bar{p}_{i^{\prime}+1}\right)=\left(\bar{p}_{i^{\prime}}-\bar{p}_{i^{\prime}+1}\right)\left(D_{i i^{\prime}}-\alpha_{i^{\prime}} \prod_{j=1}^{i^{\prime}-1}\left(1-\alpha_{j}\right)\right) \geq 0$. Also noticing that $\pi_{i}(p)$ is convex, we can conclude that $\pi_{i}(p)<\pi_{i}\left(\bar{p}_{i^{\prime}}\right), \forall p \in\left(\bar{p}_{i^{\prime}+1}, \bar{p}_{i^{\prime}}\right)$. Iteratively, we get $\pi_{i}(p) \leq \pi_{i}\left(\bar{p}_{i+1}\right), \forall p<\bar{p}_{i+1}$. For firms at position $n-1$ and $n$, clearly, it is unprofitable to price below

expected profit at the upper bound $\bar{p}_{1}$ for the firm in position 2 ,

which is incurred by the mass point in $F_{1}(\cdot)$ at $\bar{p}_{1}$. However, a jump down at one single point with zero probability measure does not affect the actual profit. $\bar{p}_{n}$, since otherwise the profit margin would decrease without increase in demand. Hence, there is no unilateral profitable deviation by pricing below the given support.

Combining (ii) and (iii), we conclude that given other firms' strategies, each firm is indifferent in pricing over the given support and has no profitable deviation. Therefore, the price strategies described in the proposition is a mixed strategy equilibrium.

\section{Proof of Proposition 2}

For $i=2, \ldots, n-1$, denote $E_{i}^{-}\left(p_{i}\right)$ and $E_{i}^{+}\left(p_{i}\right)$ as the price expectations from position $i$ conditional on $p_{i} \in\left[\bar{p}_{i+1}, \bar{p}_{i}\right]$ and conditional on $p_{i} \in\left[\bar{p}_{i}, \bar{p}_{i-1}\right]$, respectively. Also denote $f_{i}^{-}(\cdot)$ and $f_{i}^{+}(\cdot)$ as the corresponding conditional probability density functions. According to Eq.(13), both $f_{i}^{-}(p)$ and $f_{i+1}^{+}(p)$ take the form of $\frac{K}{p^{2}}$ on the same support $\left[\bar{p}_{i+1}, \bar{p}_{i}\right]$ and thus must be identical (It can also be calculated that $K$ is $\frac{\bar{p}_{i} \bar{p}_{i+1}}{\bar{p}_{i}-\bar{p}_{i+1}}$ in the former and is $\frac{\alpha_{i}}{\alpha_{i+1}\left(1-\alpha_{i}\right)} \frac{\bar{p}_{i} \bar{p}_{i+1}}{\bar{p}_{i+2}}$ in the latter. They are the same due to Eq.(2) and (3). Therefore, $E_{i}^{-}\left(p_{i}\right)=E_{i+1}^{+}\left(p_{i+1}\right)$. Notice that the expected price from the $i$ th position $E\left(p_{i}\right)$ is the weighted average of $E_{i}^{-}\left(p_{i}\right)$ and $E_{i}^{+}\left(p_{i}\right)$, and that from $i+1$ th position $E\left(p_{i+1}\right)$ is the weighted average of $E_{i+1}^{-}\left(p_{i+1}\right)$ and $E_{i+1}^{+}\left(p_{i+1}\right)$. Since $E_{i}^{-}\left(p_{i}\right)=E_{i+1}^{+}\left(p_{i+1}\right)$ and $E_{i}^{+}\left(p_{i}\right)>E_{i+1}^{-}\left(p_{i+1}\right), \quad E\left(p_{i}\right)>E\left(p_{i+1}\right)$. Similar arguments apply to position 1 and position $n$.

\section{Proof of Proposition 3}

(i) Given firms' pricing strategies, we want to show consumers' search strategies are rational. First, given $F_{1}(p)$, by Eq.(6),

$$
E G_{1}=1-(1-\ln \alpha) \alpha r \geq k>0
$$

where the first inequality is ensured by the parametric conditions on $\alpha$ and $k$. Therefore, $\sigma(0)=\sigma(k)=1$ satisfies the rational-expectations condition. Second, by substituting $F_{2}(p)$ into Eq.(7), we have

$$
\int_{\underline{p}}^{r}(r-x) d F_{2}(x)=\frac{1-\alpha+\alpha \ln \alpha}{1-\alpha} r \leq k
$$

Therefore, $r(k)=r$ satisfies the rational-expectations condition.

(ii) Given consumers' search strategy, we want to show that either firm has no unilateral profitable deviation in pricing. First, either firm achieves constant expected 
profit within the price support, because for any $p \in[\alpha r, r)$,

$$
\begin{aligned}
& \pi_{1}(p)=\alpha p+\left[1-F_{2}(p)\right](1-\alpha) p \equiv \alpha r \\
& \pi_{2}(p)=\left[1-F_{1}(p)\right](1-\alpha) p \equiv(1-\alpha) \alpha r
\end{aligned}
$$

Second, by pricing above $r$, either firm achieves zero expected profit. Similarly, by pricing below $\alpha r$, firm 1 achieves expected payoff lower than $\alpha r$ and firm 2 achieves expected payoff lower than $(1-\alpha) \alpha r$.

Altogether, the strategy profile is a rationalexpectations equilibrium.

\section{Proof of Proposition 4}

By Eq.(12), we can calculate the expected price from position $i$ according to

$$
E\left(p_{i}\right)=\bar{p}-\int_{\underline{p}}^{\bar{p}} F_{i}(p) d p
$$

so that $E\left(p_{1}\right)=(1-\ln \alpha) \alpha r$ and $E\left(p_{2}\right)=\frac{-\alpha \ln \alpha}{1-\alpha} r$. Thus, $E\left(p_{1}\right)-E\left(p_{2}\right)=(1-\alpha+\alpha \ln \alpha) \frac{\alpha}{1-\alpha} r$. It is easy to show that $1-\alpha+\alpha \ln \alpha>0$ for $\alpha<1$.

\section{References}

[1] Baye, M.R., Morgan, J. and Scholten, P. “Temporal Price Dispersion: Evidence from an Online Consumer Electronics Market," Journal of Interactive Marketing (18:4), 2004, pp. 101-115.

[2] Beatty, S. E. and Smith, S. M. "External Search Effort: An Investigation across Several Product Categories," The Journal of Consumer Research (14:1), 1987, pp. 83-95.

[3] Chen, S. and Hitt, L.M. "Measuring Switching Costs and the Determinants of Customer Retention in Internet-enabled Business: A Study of the Online Broderage Industry," Information Systems Research (13:3), 2002, pp. 255-274.

[4] Childers, T. L., Carr, C. L., Peck, J., and Carson, S. "Hedonic and Utilitarian Motivations for Online Retail Shopping Behavior," Journal of Retailing (77:4), 2001, pp. 511-535.

[5] Clay, K., Krishnan, R. and Wolff, E. "Prices and Price Dispersion on the Web: Evidence from the Online Book Industry," Journal of Industrial Economics (49:4), 2001, pp. 521-539.

[6] Diamond, P. A. "A model of Price Adjustment," Journal of Economic Theory (3:2), 1971, pp. 156-168.

[7] Ghose, A. and Yang, S. 2007. An empirical analysis of search engine advertising: Sponsored search and cross-selling in electronic markets. Forthcoming in Management Science.

[8] Hoque, A. Y. and Lohse, G. L. "An Information Search Cost Perspective for Designing Interfaces for Electronic Commerce," Journal of Marketing Research (36:3), 1999, pp. 387-394.

[9] Iyer, G., and Pazgal, A. "Internet Shopping Agents: Virtual Colocation and Competition," Marketing Science (22:1), 2003, pp. 85-106.
[10] Johnson, E. J., Moe, W. W., Fader, P. S., Bellman, S. and Lohse, G. L. "On the Depth and Dynamics of Online Search Behavior," Management Science (50:3), 2004, pp. 299-308.

[11] Smith, M.D. and Brynjolfsson, E. "Consumer Decisionmaking at an Internet Shopbot: Brand Still Matters," Journal of Industrial Economics (49:4), 2001, pp. 541-558.

[12] Stahl, D. O. 1989. Oligopolistic pricing with sequential consumer search. The American Economic Review 79(4) 700712 .

[13] Varian, H. R. "A model of sales," The American Economic Review (70:4), 1980, pp. 651-659.

[14] VSS. 2007. Veronis Suhler Stevenson Communications Industry Forecast 2007-2011. Market research report, Veronis Suhler Stevenson (VSS)

[15] Weber, T. A. and Zheng Z. 2007. A model of search intermediaries and paid referrals. Information Systems Research 18(4) 414-436.

[16] Xu, L., Chen, J. and Whinston, A. B. 2008. To place better or price cheaper? Bidding and pricing under keyword advertising. Working paper. 